\title{
Measurements and full-field predictions of deformation heterogeneities in ice
}

\author{
Maurine Montagnat ${ }^{\mathrm{a}, *}$, Jane R. Blackford ${ }^{\mathrm{b}}$, Sandra Piazolo ${ }^{\mathrm{c}}$, Laurent Arnaud ${ }^{\mathrm{a}}$, Ricardo A. Lebensohn ${ }^{\mathrm{d}}$ \\ a Laboratoire de Glaciologie et Géophysique de l'Environnement, CNRS/UJF, St Martin d'Hères, France \\ ${ }^{\mathrm{b}}$ Institute for Materials and Processes, School of Engineering, The University of Edinburgh, Edinburgh, UK \\ c University of Stockholm, Stockholm, Sweden, presently at: GEMOC ARC National Key Centre, Department of Earth and Planetary Sciences, Macquarie University, Sydney, Australia \\ ${ }^{\mathrm{d}}$ Materials Science and Technology Division, Los Alamos National Laboratory, Los Alamos, NM, USA
}

\begin{abstract}
We have made creep experiments on columnar grained ice and characterised the microstructure and intragranular misorientations over a range of length scales. A FFT full-field model was used to predict the deformation behaviour, using the experimentally characterised microstructure as the starting material. This is the first time this combination of techniques has been used to study the deformation of ice. The microstructure was characterised at the $\mathrm{cm}$ scale using an optical technique, the automatic ice texture analyser AITA and at the micron scale using electron backscattered diffraction EBSD. The crystallographic texture and intragranular misorientations were fully characterised by EBSD ( 3 angles). The deformed microstructure frequently showed straight subgrain boundaries often originating at triple points. These were identified as kink bands, and for the first time we have measured the precise misorientation of the kink bands and deduced the nature of the dislocations responsible for them. These dislocations have a basal edge nature and align in contiguous prismatic planes enabling deformation along the c-axis. In addition, non-uniform grain boundaries and regions of recrystallization were seen. We present coupling between fine scale characterization of intragranular misorientations, from experiments, and prediction of internal stresses that cause it. The model predicts the morphology of the observed local misorientations within the grains, however it over predicts the misorientation values. This is because the annealing and recrystallization mechanisms are not taken into account in the model. Ice is excellent as a model material for measuring, predicting and understanding deformation behaviour for polycrystalline materials. Specifically for ice this knowledge is needed to improve models of ice sheet dynamics that are important for climatic signal interpretation.
\end{abstract}

\section{Introduction}

To improve climatic signal interpretation, and thus predictions, accurate modelling of ice dynamics is essential. In particular, an understanding of the deformation and recrystallization processes is necessary to correctly represent the ice flow in ice sheet modelling (Castelnau et al., 1996b; Durand et al., 2007). Indeed, to interpret the climatic signal correctly, dating of the ice core strongly relies on ice flow models, available ice-age markers, and ice texture characterization as a marker of ice flow discontinuities (Buiron et al., 2011; Greenland Ice core Project (GRIP) Members, 1993; Parrenin et al., 2001).

Ice is an hexagonal material in which deformation mainly occurs by dislocation glide along the basal plane conferring a strong viscoplastic anisotropy to the crystal (Duval et al., 1983). Such anisotropy at the crystal scale induces the development of strong internal stresses during deformation of the polycrystal, associated with the mismatch of dislocation slip between neighbouring grains. This internal state of

\footnotetext{
* Corresponding author.

E-mail address: montagnat@lgge.obs.ujf-grenoble.fr (M. Montagnat).

URL: http://www-lgge.obs.ujf-grenoble.fr/maurine/maurine.html (M. Montagnat).
}

stress strongly influences the deformation behaviour and recovery mechanisms such as dynamic recrystallization. Dynamic recrystallization mechanisms are very efficient in ice (Duval, 1979; Jacka and Li, 1994; Kipfstuhl et al., 2006; Montagnat et al., 2009). They are known to accommodate deformation processes as observed along ice cores taken from ice sheets (Alley et al., 1986a,b; de la Chapelle et al., 1998; Duval and Castelnau, 1995; Montagnat and Duval, 2000) and to influence the texture evolution and thus the flow of ice.

Metals, rocks and ice, all polycrystalline aggregates, show remarkable similarities in their deformation and recrystallization behaviour (see for instance Frost and Ashby, 1982; Goodman et al., 1981; Humphreys and Hatherly, 1996; Kocks et al., 1998; Schulson and Duval, 2009). In this respect, both ice core data and ice deformation laboratory experiments provide very good systems to study the deformation heterogeneity development and dynamic recrystallization mechanisms for highly anisotropic materials. As such they constitute a valuable data set to validate modelling approaches for polycrystal mechanical behaviour (Castelnau et al., 1996b, 1997; Gilormini et al., 2001; Lebensohn et al., 2007).

We present a detailed study of the characterization of deformation heterogeneities and link these with the internal stress development 
during the creep of ice. We place particular emphasis on frequently observed kink bands in relation to the nature of the dislocations involved, and strain accommodated.

Laboratory experiments with columnar grained ice were used to quantify the intragranular misorientations that appear at the end of transient creep, and are considered as precursors to recrystallization mechanisms. An Automatic Ice Texture Analyser (AITA) (Russell-Head and Wilson, 2001) provides c-axis rotation measurements at the full sample scale (up to $120 \times 120 \mathrm{~mm}^{2}$ ), and electron backscatter diffraction (EBSD) measures the full crystal orientation data at the grain scale following Piazolo et al. (2008).

Although common in metallic materials, such detailed local characterization of misorientations is at present not commonly performed in ice. For ice, most observations of local misorientations have been qualitative (Hamman et al., 2007; Mansuy et al., 2000, 2002; Wilson and Zhang, 1994), or obtained with X-ray diffraction techniques which do not allow mapping of a full area within the sample (Montagnat et al., 2003; Weikusat et al., 2011). Previous EBSD measurements were performed mainly to provide fabric data on the samples extracted along ice cores, with little consideration of local misorientations (Obbard and Baker, 2007; Obbard et al., 2006).

In order to predict quantitatively the internal state of stress and strain rate responsible for the observed intragranular misorientations we use the FFT full-field viscoplastic modelling approach (Lebensohn, 2001). Although this has already been validated for ice (Lebensohn et al., 2009), here, for the first time, we extend the methodology by using direct input from experimental measurements.

\section{Experimental procedure and analyses}

Experiments were carried out on laboratory grown ice samples with columnar grains in the direction perpendicular to the compression axis. Laboratory grown columnar ice is an excellent model material as its starting grain structure is well defined, near "2D" and without heterogeneities such as bubbles or impurities.

The sample dimensions were $50 \times 50 \times 10 \mathrm{~mm}^{3}$, with an average grain diameter of about $10 \mathrm{~mm}$. The specimens were deformed in a cold room at $-11^{\circ} \mathrm{C}+/-1{ }^{\circ} \mathrm{C}$ using a mechanical press with a level arm to apply uniaxial compression at a constant load of $0.5 \mathrm{MPa}$. The compression was applied perpendicular to the long axes of the columnar grains (see Fig. 1).

The test was stopped at about $2 \%$ strain, close to the minimum creep rate which ensured the test ended before substantial recrystallization

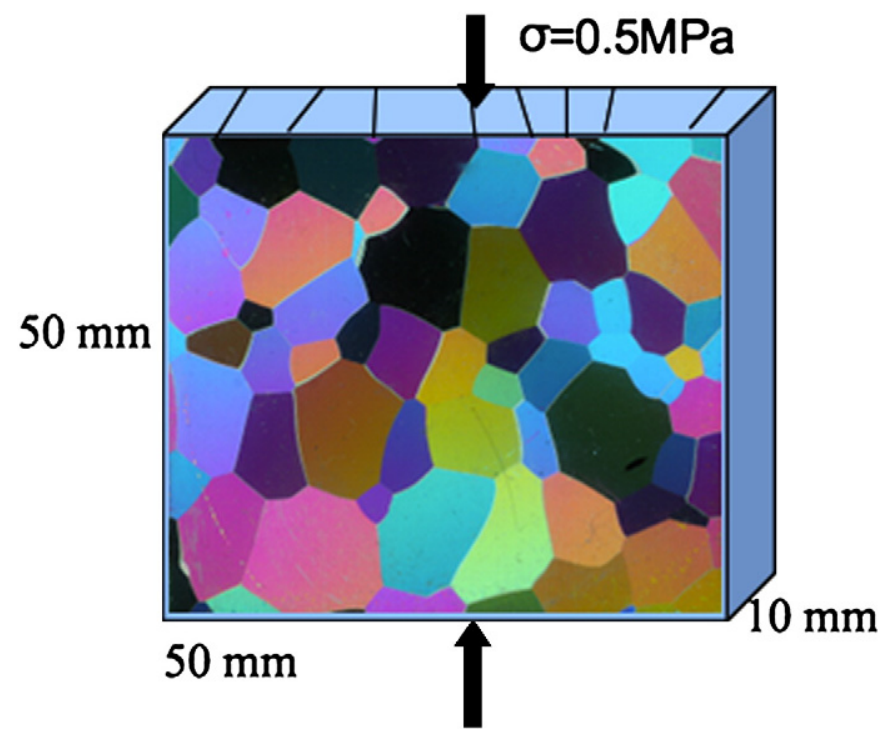

Fig. 1. Schematic representation of the experimental condition for the creep test. occurred (Jacka and Li, 1994). The strain rate at the end of the test was about $1.5 \times 10^{-7} s^{-1}$, which is comparable to that reported by Ple and Meyssonnier (1997) for similar creep test on columnar ice. Our samples with "2D" geometry contain only few grains, and one grain in the thickness. The strain rates measured during our creep test are in the middle range between the granular ice and the single crystal behaviour. Although limited experimental work has been done with columnar ice the viscosity is expected to be lower than that of the granular ice, and the stress exponent is expected to be 3 in our experimental conditions (see Schulson and Duval (2009) for a review of the results available).

The Automatic Ice Texture Analyser (Russell-Head and Wilson, 2001) provides the c-axis orientations from an ice thin section (i.e. $\approx 0.25 \mathrm{~mm}$ of ice fixed on a glass plate) of the whole specimen, with a spatial resolution of $43 \mu \mathrm{m}$ and an angular resolution of $+/-1^{\circ}$. The c-axis orientation data are given via the azimuth and colatitude values.

EBSD analysis was performed on a Philips XL-30-ESEM-FEG at the Department of Geological Sciences at Stockholm University. Samples analysed were uncoated. They were frozen to the pre-tilted sample holder sledge and during EBSD analyses the ice was maintained at a temperature of -90 to $-100{ }^{\circ} \mathrm{C}$ using the Gatan cold stage C1001 Cooling Stage Module with tilted sample holder for EBSD (for more details see Piazolo et al. (2008)). Data were collected by moving the beam across a rectangular area at steps of $3 \mu \mathrm{m}$. Noise reduction was performed following Prior (1999). In the following, EBSD data are represented in different ways. Orientation contrast images show a combination of surface topography and crystallographic orientation. Textural component maps show the difference in angle of each point analysis relative to a chosen reference orientation (marked with a cross). In addition, pole figures (equal area, upper hemisphere) are used to represent the statistical orientation dispersion. In all analyses the same $X Y Z$ sample coordinate system is used where $Z$ is out of plane and the $\mathrm{X}$ direction is parallel to the main compression axis.

\section{Substructure characterization: subgrain boundaries, local misorientations and type of dislocations}

Fig. 2 shows the c-axis orientation image from the sample after the compression test, along with two azimuth profiles from selected representative areas (profiles along the two thick black lines).

The observations performed with the AITA at the sample scale reveal that a strong localisation of misorientations develops close to triple junctions and grain boundaries. In addition, at grain boundary asperities, misorientations including discontinuous subgrain boundaries and occasionally small recrystallized grains are seen (labelled RX in Fig. 2).

The deformed microstructure is dominated by straight subgrain boundaries which initiate at triple junctions and commonly develop parallel to the initial c-axis orientation of the grain (arrows in Fig. 2). These boundaries represent short distance (at the sample scale) reversed misorientations with sharp and strongly localised misorientations (see profile 1 in Fig. 2). Misorientations were also found to result from a progressive rotation of the c-axis over the grain (profile 2 in Fig. 2). In both cases, they accommodate c-axis misorientations of several degrees. Note that since the AITA only measures c-axis orientations, shear bands, which do not induce any c-axis misorientation cannot be seen in Fig. 2 but were frequently observed in previous studies (Mansuy et al., 2000, 2002).

EBSD analyses were performed to investigate the main intracrystalline features observed at a higher resolution and with complete crystallographic data (both c- and a-axis orientations). Figs. 3 and 4 present one of the areas selected to investigate the nature of the straight subgrain boundaries visible in Fig. 2. This area is located along the boundary between grains A and B of Fig. 2. Due to sample configuration, Fig. 3 represents this area up side down and back to front compared to the representation of Fig. 2. 

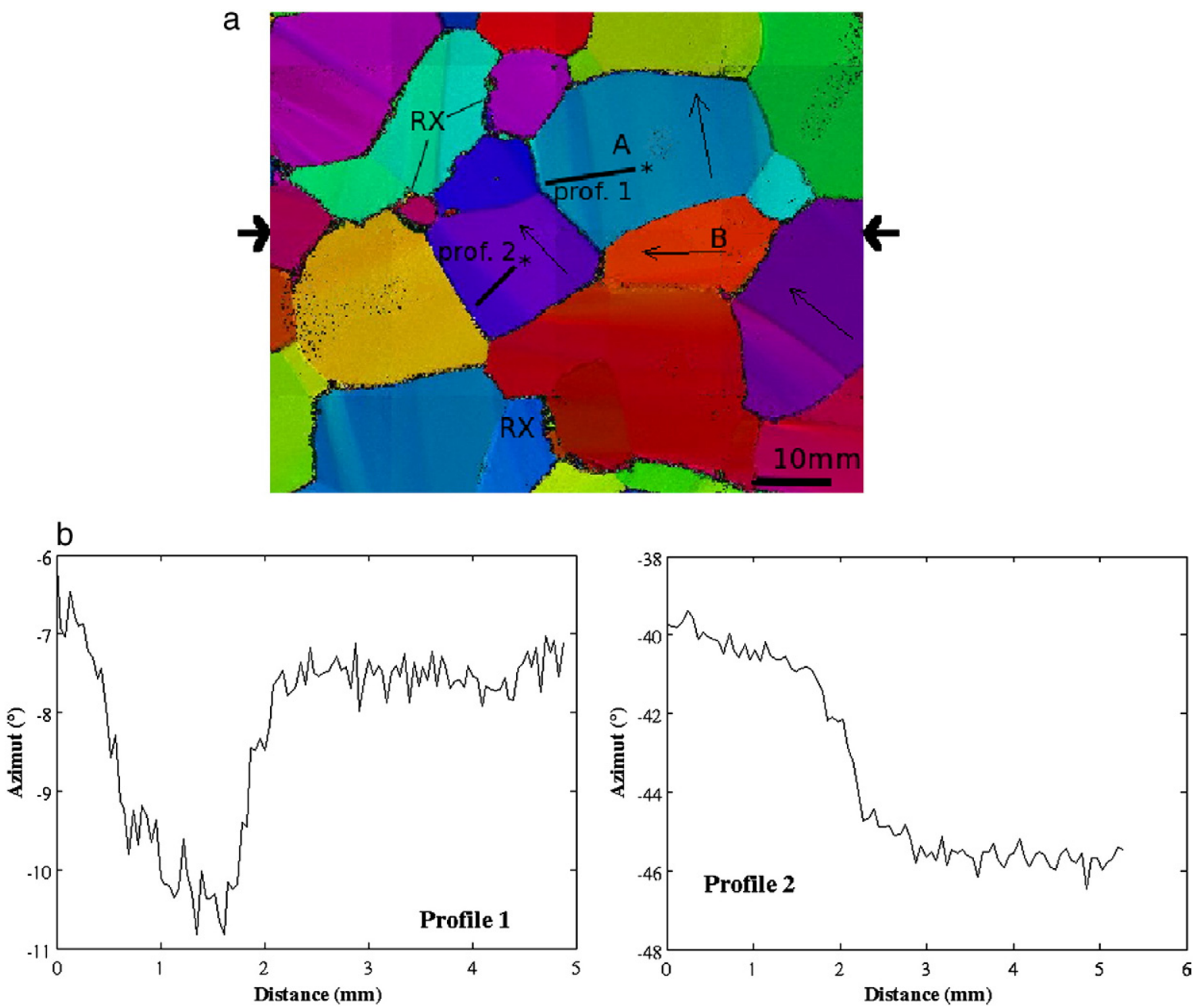

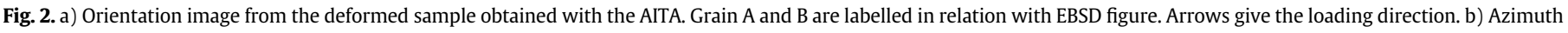

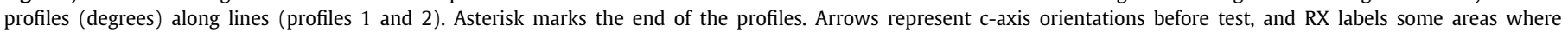
recrystallization was observed.

As shown in Fig. 3b), the change in orientation between the straight subgrains appearing near the triple junction reveals a nearly perfect kink band structure, where two subgrains with very similar orientation are separated by a subgrain with significantly different orientation. Fig. 4 a) and b) are the pole figures extracted from grains $A$ and $B$ respectively. The observed continuous small circle dispersions of orientations are explained by a plastic strain of the lattice controlled by dislocations (Lloyd, 1991; Lloyd and Freeman, 1994; Prior, 1999; Prior et al., 2002). These dislocations are arranged in distinct subgrain boundaries in which dislocations of a particular geometry form dislocation arrays creating a misorientation with a specific orientation and rotation axis within the crystal.

Fig. 3a) also shows a high quantity of small groove lines of irregular shape emerging from grain boundary asperities. As illustrated in Fig. 5, these groove lines are distinct low angle subgrain boundaries which are defined as a change in orientation from one side to the other side of the boundary. Observed orientation changes are in the range of 1.5 to $10^{\circ}$ and separate areas of like orientation i.e. subgrains (Fig. 5). The number of groove lines observed is higher close to triple junctions. Such intragranular misorientations must be associated with a high and irregular local level of dislocations on both sides of grain boundaries. Such an irregularly stored strain energy induces a local and irregular grain boundary migration that results in the grain boundary irregularities observed after the test (Figs. 2 and 3).
In order to define the most likely activated slip systems, boundary type and type of dislocations involved, we used the boundary trace technique suggested by Prior et al. (2002). This technique relies on the identification of the rotation axis and the measured orientation of the subgrain boundary in the plane of observation. Rotation axis denotes the crystallographic direction shared by the two adjacent subgrains while all other crystallographic directions show a slight mismatch in terms of orientation. Consequently, such a rotation axis can be identified as the axis along which there is no orientation change across the boundary. In the case of a tilt boundary which is formed by edge dislocations the rotation axis must lie in the plane of the subgrain boundary, while for a twist boundary formed by screw dislocations the rotation axis is perpendicular to the subgrain boundary plane (for graphical representation see Reddy et al. (2007)).

Subgrain boundaries observed in deformed samples show a lack of orientation dispersion along one of the $<1010>$ axes (Fig. 4). At the same time, these rotation axes are impossible or very unlikely to be lying perpendicular to the boundary plane which is likely to be close to perpendicular to the sample surface. In contrast, the rotation axes can easily lie within the boundary plane which is consistent with the boundary being a tilt boundary formed by the alignment of edge dislocations.

These analyses lead us to the conclusion that the observed intragranular misorientations, from small subgrain boundaries, dislocation arrays or kink bands, are mainly associated with basal 
a

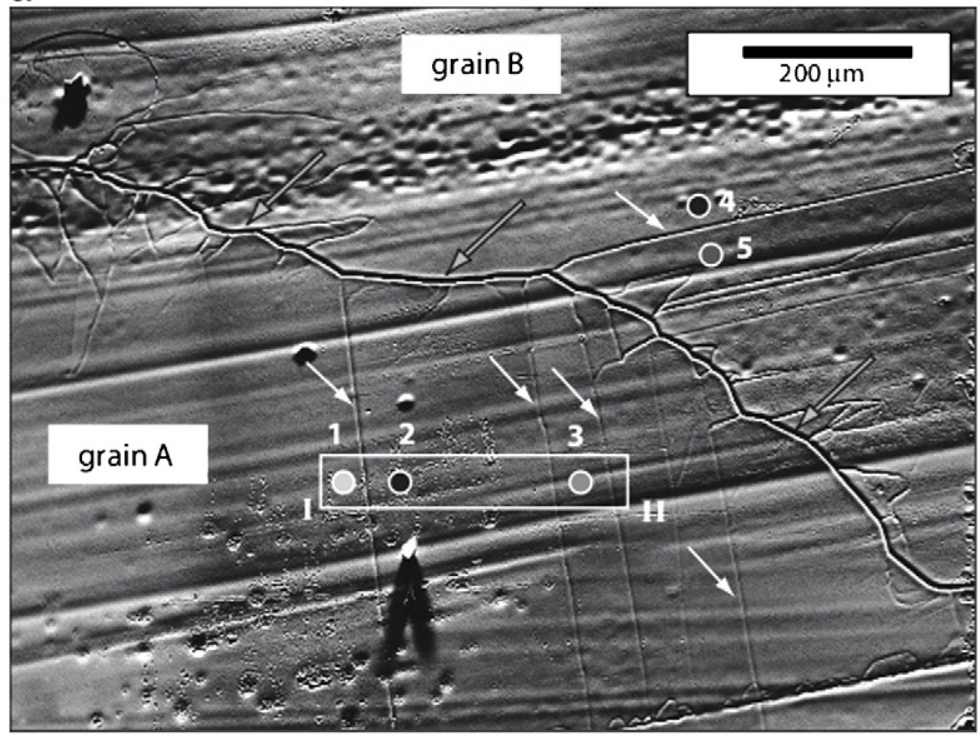

b

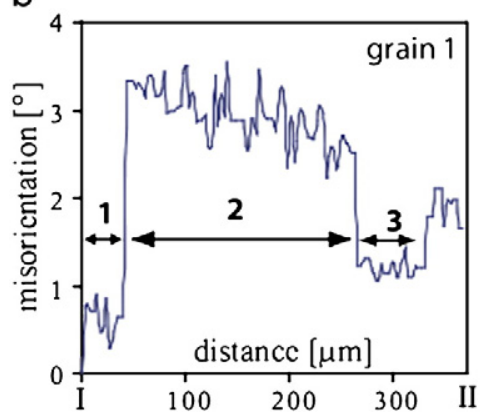

C

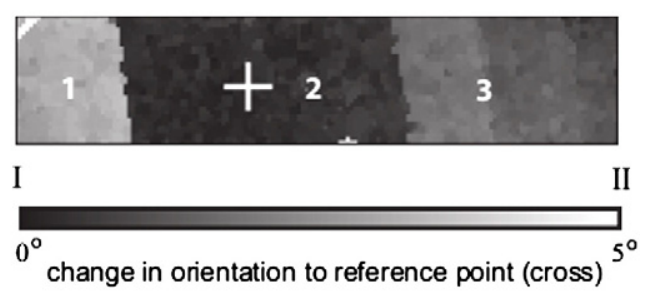

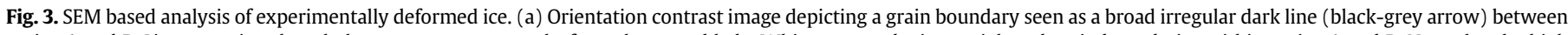

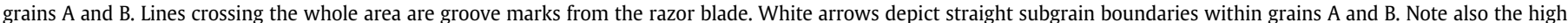

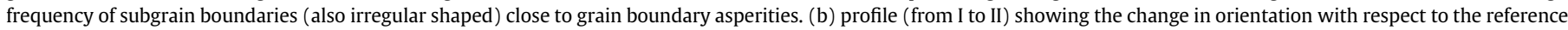

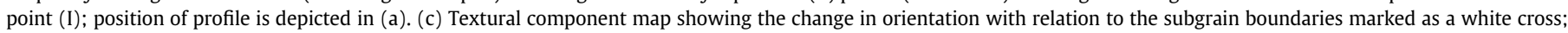
position of EBSD map shown in (a).

edge dislocations. In the kink bands, edge dislocations form arrays in contiguous prismatic planes, creating tilt boundaries, which confirms preliminary observations from Piazolo et al.(2008).

The observed straight boundaries with systematic reversal of misorientation have qualitatively been described as kink bands by Mansuy et al. (2000, 2002), Wilson and Zhang (1994), Wilson et al. (1986). In the early Orowan (1942) description, kink boundaries consist of planes which bisect the angle between the glide planes on either side of them, and along which the dislocations are concentrated. The precise misorientation measurement performed here confirms that the observations are highly compatible with such a representation, as illustrated by profile 1 of Fig. 2 at the grain scale, and by local crystallographic orientation measurements provided by the EBSD analyses (Fig. 3). In the ideal model, the orientations on both sides of the kink band are the same. At the grain scale (Fig. 2), the observed discrepancy could be attributed to the low accuracy of the observations which do not enable deconvolution of the contributions of several sub-structure misorientations. Within these contributions continuous misorientation following the kink band should appear, with dislocations arranged in arrays. With better resolution from EBSD measurements, the kink band structure clearly appears and is explained by the contribution of edge dislocations trapped in arrays.

The kink bands observed in ice are clearly related to localised plasticity as shown by our EBSD analyses, with a key role attributed to basal dislocations. In our study, most of the observed kink bands, are localised in grains where basal glide is facilitated as seen by Mansuy et al. (2000). Such a configuration is then close to the type II kink bands observed in quartz by Nishikawa and Takeshita (1999) which were shown to form at a low stress level, when the anisotropic plane is inclined to the compression axis. Kink band formation as observed and quantified in this study clearly participates to the deformation along the c-axis imposed by a highly heterogeneous state of stress, mostly concentrated at grain boundaries and triple junctions.

\section{Prediction of the local state of stress responsible for deformation heterogeneities}

We used the full-field FFT-based formulation, originally proposed by Moulinec and Suquet (1998) and adapted to compute the local response of viscoplastic anisotropic 3D polycrystals by Lebensohn (2001), to investigate the intragranular state of stress and strain rate responsible for the formation of the local misorientations and kink bands observed in our experiments.

The viscoplastic FFT-based formulation consists in finding a strainrate field, associated with a kinematically-admissible velocity field, which minimises the average of local work-rate, under the compatibility and equilibrium constraints. The method is based on the concept that the local mechanical response of a periodic heterogeneous medium can be calculated as a convolution integral between 

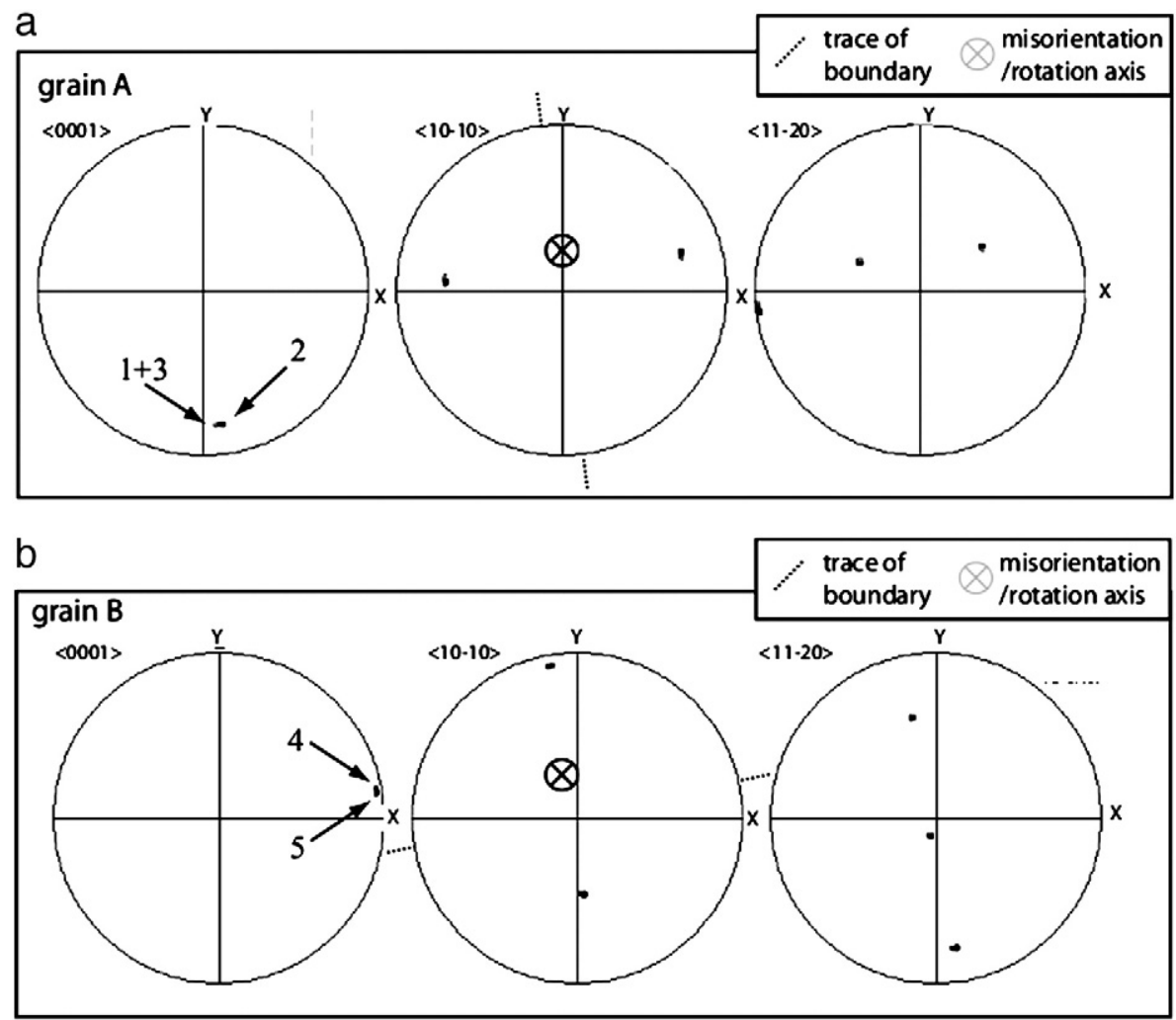

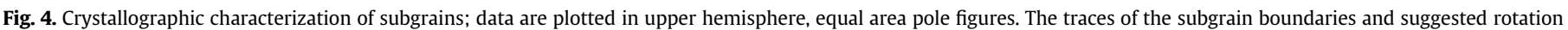
axes are depicted. (a) Grain A subgrains (1-3), and (b) grain B subgrains (4 and 5).
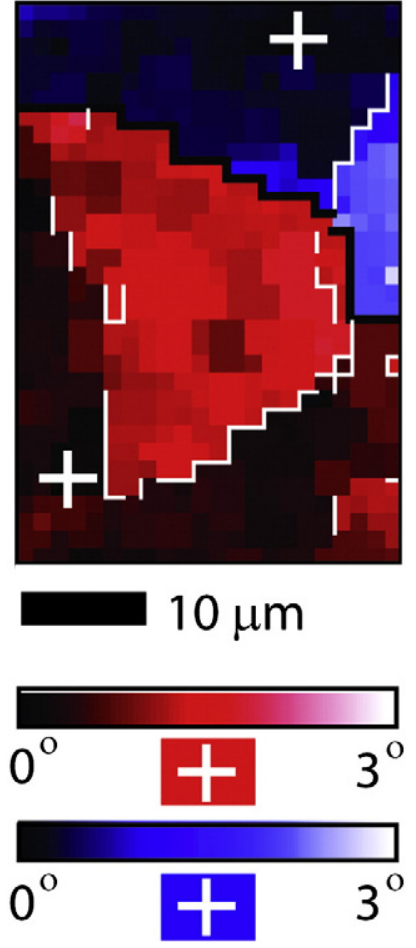

Fig. 5. Presence of subgrain boundaries and distinct local orientation variations close to a grain boundary: here textural component maps from two adjacent grains are shown. Boundaries with more than $10^{\circ}$ misorientation i.e. grain boundary are depicted as black lines, and $1-10^{\circ}$ boundaries as white lines. The image illustrates in colour scale the variation in orientation with respect to reference points on both sides of the grain boundary (white crosses). the Green function of a linear reference homogeneous medium and the actual heterogeneity field. The detailled formulation will not be described here, but can be found in (Lebensohn, 2001; Lebensohn et al., 2009) for instance.

The FFT-based formulation was recently applied to compute the internal state of stress and strain rate in polycrystalline ice with artificial Voronoi microstructure (Lebensohn et al., 2009). In the present work, we used the microstructure and orientation data obtained from AITA measurements on the sample as the input to the $2 \mathrm{D}$ version of the code. The application of the FFT formulation requires the generation of a periodic unit cell or representative volume element (RVE). The experimental microstructure, discretized into $1024 \times 1024$ Fourier points, constitutes the RVE.

The input data requires the knowledge of all three Euler angles $\left(\phi_{1}\right.$, $\theta$, and $\left.\phi_{2}\right)$. In the $2 \mathrm{D}$ restriction, $\theta$ is equal to $90^{\circ}, \phi_{1}$ is the projection of the measured c-axis orientation in the plane, and $\phi_{2}$ is randomly chosen. In order to satisfy the required periodic conditions, border grains were reproduced on opposite sides without noticeably modifying the original microstructure.

An average velocity gradient is imposed on the unit cell, which can be decomposed into an average strain-rate and an average rotationrate. For each point of the grid, the local constitutive relation between the strain-rate and the deviatoric stress is given by the classic incompressible rate-dependent crystal plasticity equation, by adding the contribution to deformation of the 12 slip systems supposed to be active in ice (Castelnau et al., 1996a, 1997; Lebensohn et al., 2004). These slip systems are traditionally 3 basal, 3 prismatic and 6 pyramidal systems. The relative activation of each slip system is controlled by the critical resolved shear stress parameter fixed initially for each system. Values used here render the basal slip system twenty times easier to activate than the prismatic and pyramidal ones (Lebensohn et al., 2009). 
From the anti-symmetric Schmid tensor, the rotation rate of the crystallographic lattice is calculated for each point in the material. This rotation rate is used to determine the orientation evolution during the test.

The following strain-rate tensor was imposed up to $2 \%$ strain

$$
\left(\begin{array}{ccc}
10^{-7} s^{-1} & 0 & 0 \\
0 & 10^{-7} s^{-1} & 0 \\
0 & 0 & 0
\end{array}\right)
$$

Note that our analysis was restricted to the local fields obtained for a fixed configuration, corresponding to the secondary creep state obtained experimentally. In this sense, the high strain-rate regions predicted by the model (see below) should be regarded as precursors of localisation bands.

Fig. 6 represents the computed equivalent strain-rate field normalised with respect to the average equivalent strain rate $\dot{E}_{e q}=1.15 \times 10^{-7} s^{-1}$, the equivalent stress, normalised with respect to the average equivalent stress, the relative basal activity and the misorientation of the $\phi_{1}$ Euler angle relative to the initial value, for the entire unit cell after simulation (see Lebensohn et al. (2009) for details). Fig. 7 represents the $\phi_{1}$ Euler angle and azimuth values taken along the profile. This profile can be directly compared with the experimental profile obtained in zone A of Fig. 2.
Figs. 6 and 7 clearly show that this approach represents the localisation bands well, with evidence of strain concentration parallel to the c-axis. The local misorientations measured experimentally are also represented well with a clear initiation at triple junction (see Fig. 2 for comparison). Nevertheless, the amplitude of the c-axis misorientation (see Fig. 7) is generally higher than that of the measured one. This is easily explained as annealing mechanisms such as a local dynamic recrystallization through grain nucleation or local grain boundary migration are not included in this full-field scheme (a grain boundary is represented as a simple orientation transition). The modelled internal stresses and Euler angle misorientations are then upper bound values. Nevertheless, grain boundaries and triple junctions are clearly the areas with higher levels of stress concentration, where misorientations and non-basal activity concentrate.

In Fig. 6a) deformation bands parallel to the basal planes appear. Such deformation bands represent shear bands where deformation occurs by dislocation glide in the basal planes. They are generally associated with a high amount of basal activity. As mentioned before, these shear bands could not be seen in this study due to the limitations of the orientation measurement tool, but were observed by (Mansuy et al., 2000, 2002).

The predicted internal stresses can be more than five times the macroscopic equivalent stress around areas where deformation and local misorientations concentrate. To accommodate these internal a

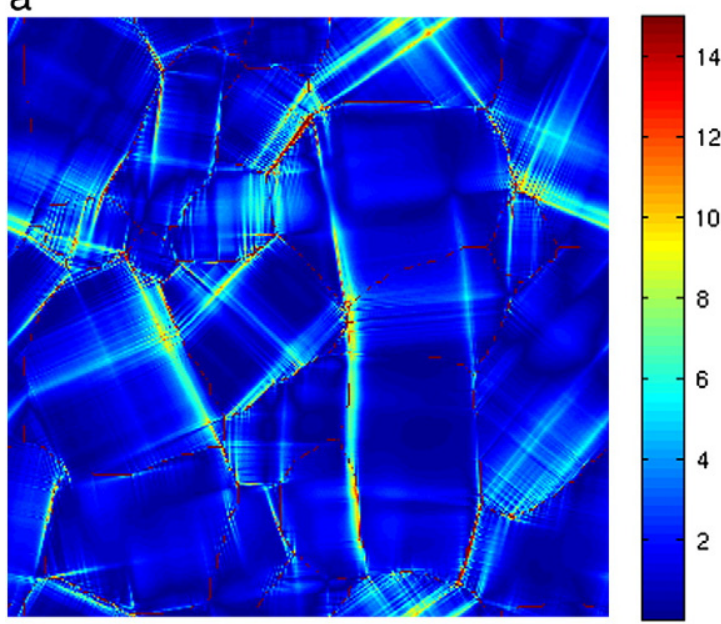

C

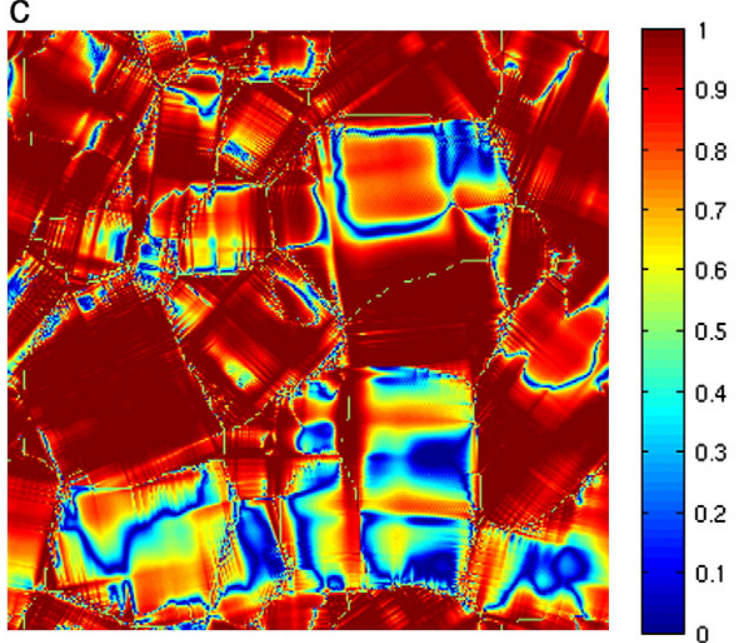

b

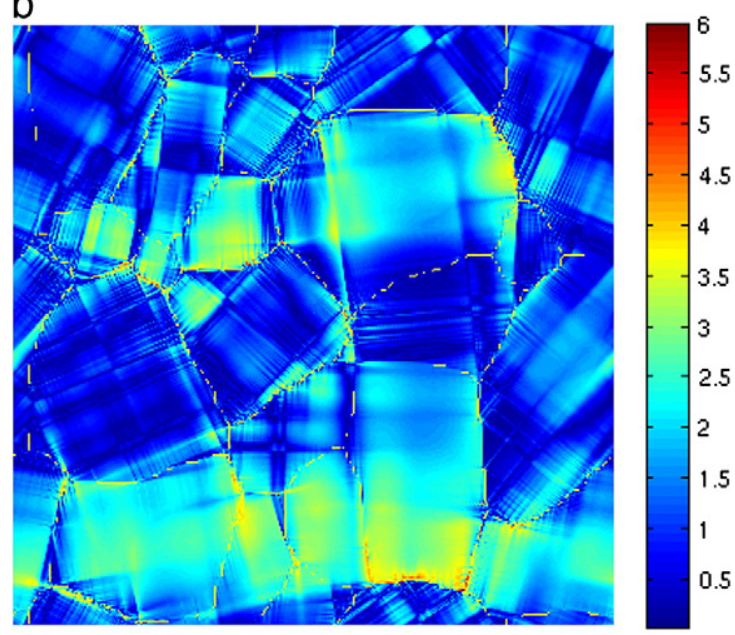

d

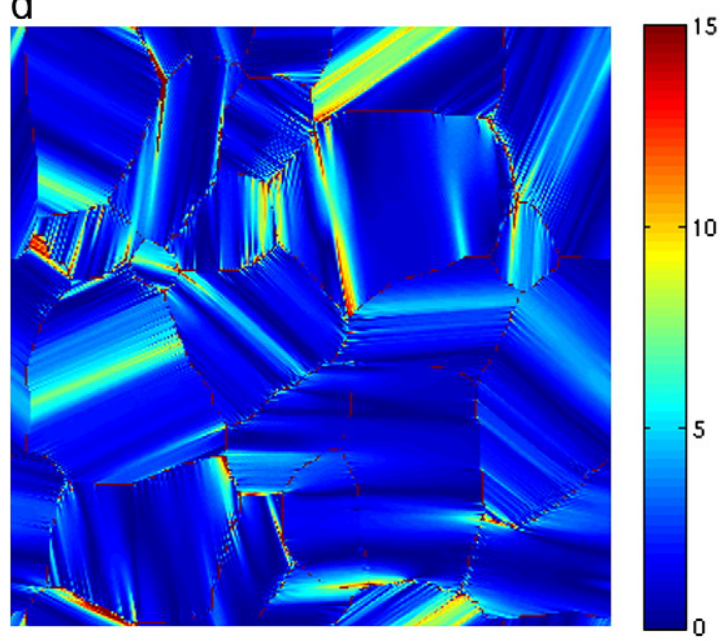

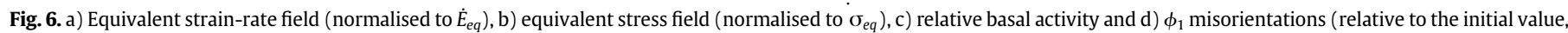
in degrees) obtained over the unit cell with the FFT full-field modelling. 
a
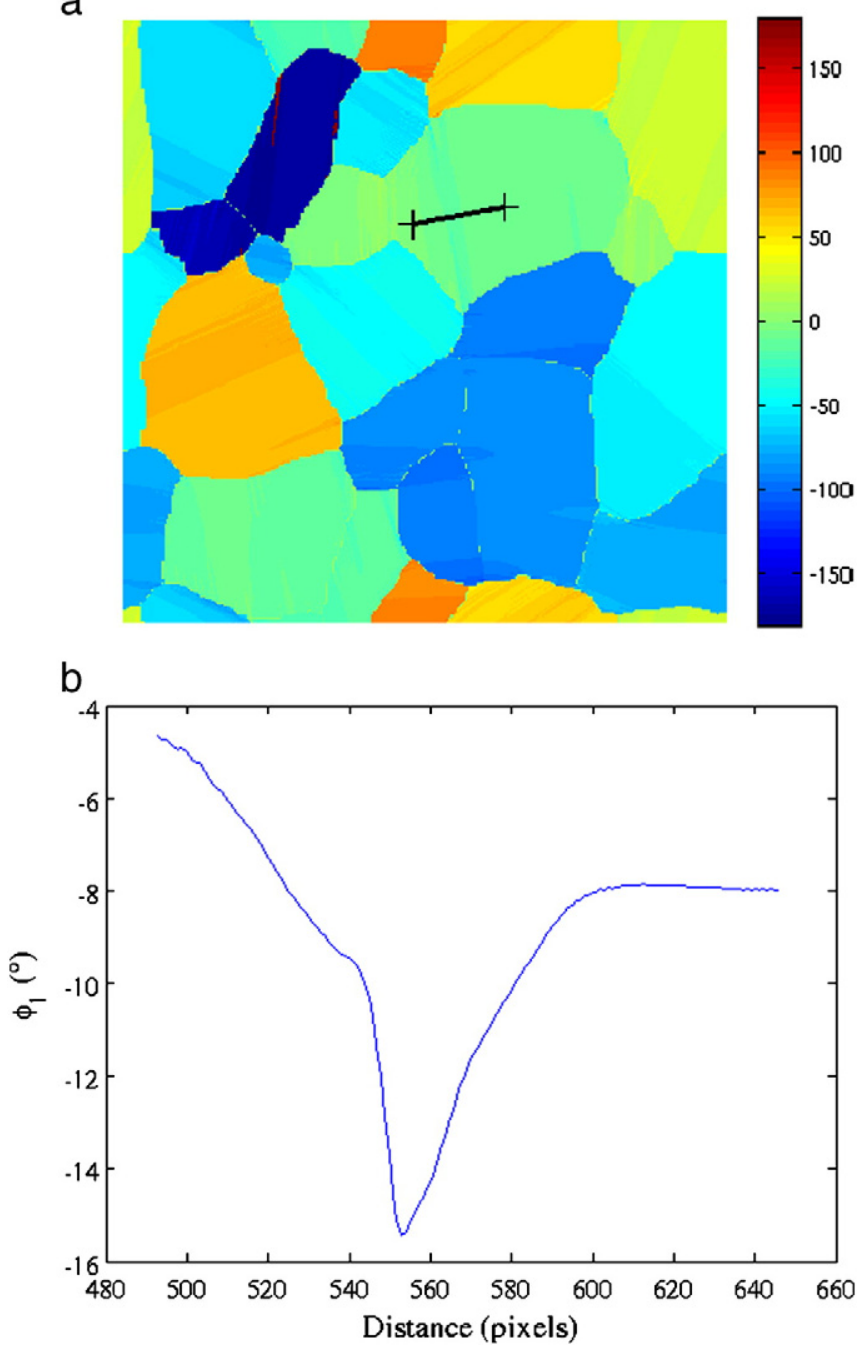

Fig. 7. Left: $\phi_{1}$ Euler angle after deformation, from FFT full-field modelling. Right: $\phi_{1}$ Euler angle profile in degrees obtained along the black line, from left to right.

stresses, a local high level of non-basal activity is required. This nonbasal activity, as shown by Lebensohn et al. (2009), is mostly due to the activity of pyramidal slip systems providing the required deformation along the c-axis and remains low in average over the whole sample (less than 15\%). It is worth noting that due to the planestrain condition and the in-plane orientation of the c-axes, the prismatic slip systems are not well oriented for any $\phi_{1}$ angle.

The model clearly predicts a strong correlation between local internal stresses, high lattice misorientation and strong non-basal activity. This non-basal activity is required within the model formulation to provide the deformation along the c-axis, as produced by the kink band formation. More generally, such a non-basal activity is required in the simulation of ice viscoplastic deformation to reproduce the role of climb, diffusion or cross slip that can not be directly taken into account in this modelling approach (Ashby and Duval, 1985; Chevy et al., 2010; Montagnat et al., 2006).

\section{Concluding remarks}

Experimental observations from the c-axis orientation measurements at the sample scale (from AITA), and the full lattice misorientation analyses in selected areas (from EBSD) clearly show a high level of local misorientations close to grain boundaries, and particularly at triple junctions. A specific focus was on some straight subgrain boundaries crossing grains from triple junctions that occurred frequently. The characterization performed at these two different scales demonstrates that these subgrain boundaries are kink bands parallel to the c-axis. For the first time in ice, EBSD measurements show the basal edge nature of dislocations creating the kink bands, by aligning in contiguous prismatic planes. The observed kink bands in ice are similar to the type II kink bands described for quartz by Nishikawa and Takeshita (1999), as they are mostly created in grains where basal slip is activated. They are required to create a deformation along the c-axis when imposed by a highly heterogeneous state of stress.

The full-field modelling approach developed by Lebensohn (2001) was applied to the experimental microstructure, to predict the local state of stress responsible for deformation heterogeneities evidenced by the observation of strong lattice misorientations. Local stress values of up to five times the macroscopic equivalent stress are predicted at triple junctions where kink bands appear. Such a high local stress state is associated with the activation of a high amount of pyramidal slip, in order to reproduce the required deformation along the c-axis.

Experimental observations clearly show the initiation of local recrystallization mechanisms (grain nucleation). Recrystallization mechanisms, which strongly reduce the stress field, are not taken into account in the modelling approach, the predicted state of stress and strain rate must then be considered as an upper bound. In particular, for deformation conditions in natural polar ice sheets, the strain rate and stresses are so low that recrystallization mechanisms are supposed to strongly reduce the internal stress field. Very few kink bands have been observed so far in natural ice.

The combination of experimental and numerical techniques we have applied to ice illustrates the potential of the FFT full-field modelling approach to predict the internal state of stress and the areas where misorientations localise as a function of the neighbouring environment. Indeed, a coupling between the FFT full-field approach and the microstructure evolution model Elle is under progress to represent the recrystallization mechanisms (Piazolo et al., 2010). Ice is a good material to use for improving the modelling technique as it is relatively straightforward to make controlled mechanical tests on simple microstructures. In particular, the " $2 \mathrm{D}$ " microstructure is particularly well adapted to perform surface observations with little influence of the third dimension microstructure. We expect stress heterogeneities to be lower in a granular material, mainly because of a higher number of neighbours.

Although our experiments were made on columnar ice, the results and techniques developed are widely applicable to other polycrystalline materials, as their behaviour is based on the same underlying physics. These other materials include geological materials such as deforming mantle consisting of polycrystalline olivine (Castelnau et al., 2010; Tommasi et al., 2009) and industrial materials such as thermomechanical processing of commercial metallic alloys (see for instance (Al-Samman and Gottstein (2008), Soula et al. (2009))).

Data extracted from such an experiment/modelling coupling approach will be used to determine the effect of (i) interaction of neighbouring grains on the amplitude of local stress and strain-rate values, and (ii) recrystallization on the amplitude of internal stress evolution during deformation.

\section{Acknowledgements}

We acknowledge Albert Griera for the help in providing the initial model microstructure input, Gaël Durand for helping with the output toolbox, Paul Duval, Chris Hall and Michael Zaiser for the fruitful discussions and perceptive comments. Financial support by the Swedish Research Council (VR 621-2004-5330), the Knut and AliceWallenberg Foundation (financing of equipment), department 
INSIS of CNRS, France, and the Royal Society (joint project grant University of Edinburgh and LGGE Grenoble) are acknowledged. This contribution has been financially supported by the European Science Foundation (ESF), EUROCORES Programme EuroMinScl, FP6.

\section{References}

Al-Samman, T., Gottstein, G., 2008. Dynamic recrystallization during high temperature deformation of magnesium. Mater. Sci. Eng., A 490, 411-420.

Alley, R.B., Perepezko, J.H., Bentley, C.R., 1986a. Grain growth in polar ice: I. theory. J. Glaciol. 32, 415-424.

Alley, R.B., Perepezko, J.H., Bentley, C.R., 1986b. Grain growth in polar ice: II. application. J. Glaciol. 32, 425-433.

Ashby, M.F., Duval, P., 1985. The creep of polycrystalline ice. Cold Reg. Sc. Technol. 11, 285-300.

Buiron, D., Chappellaz, J., Stenni, B., Frezzoti, M., Baumgartner, M., Capron, E., Landais, A., Lemieux-Dudon, B., Masson-Delmotte, V., Montagnat, M., Parrenin, F., Schilt, A. 2011. TALDICE-1 age scale of the Talos Dome deep ice core, East Antarctica. Climate Past. 7, 1-16.

Castelnau, O., Canova, G.R., Lebensohn, R.A., Duval, P., 1997. Modelling viscoplastic behavior of anisotropic polycrystalline ice with a self-consistent approach. Acta Mater. 45, 4823-4834.

Castelnau, O., Cordier, P., Lebensohn, R., Merkel, S., Raterron, P., 2010. Microstructures and rheology of the earth's upper mantle inferred from a multiscale approach. C.R. Phys. 11, 304-315 Computational metallurgy and scale transitions..

Castelnau, O., Duval, P., Lebensohn, R.A., Canova, G., 1996a. Viscoplastic modeling of texture development in polycrystalline ice with a self-consistent approach : comparison with bound estimates. J. Geophys. Res. 101, 13,851-13,868.

Castelnau, O., Thorsteinsson, T., Kipfstuhl, J., Duval, P., Canova, G.R., 1996b. Modelling fabric development along the GRIP ice core, central Greenland. Ann. Glaciol. 23, 194-201.

de la Chapelle, S.D.L., Castelnau, O., Lipenkov, V., Duval, P., 1998. Dynamic recrystallization and texture development in ice as revealed by the study of deep ice cores in Antarctica and Greenland. J. Geophys. Res. 103, 5091-5105.

Chevy, J., Fressengeas, C., Lebyodkin, M., Taupin, V., Bastie, P., Duval, P., 2010. Characterizing short-range vs. long-range spatial correlations in dislocation distributions. Acta Mater. 58, 1837-1849.

Durand, G., Gillet-Chaulet, F., Svensson, A., Gagliardini, O., Kipfstuhl, S., Meyssonnier, J., Parrenin, F., Duval, P., Dahl-Jensen, D., Azuma, N., 2007. Change of the ice rheology with climatic transitions. Implication on ice flow modelling and dating of the EPICA Dome C core. Climates Past. 3, 155-167.

Duval, P., 1979. Creep and recrystallization of polycrystalline ice. Bull. Minér. 102, 80-85

Duval, P., Ashby, M., Anderman, I., 1983. Rate controlling processes in the creep of polycrystalline ice. J. Phys. Chem. 87, 4066-4074.

Duval, P., Castelnau, O., 1995. Dynamic recrystallization of ice in polar ice sheets. J. Phys. IV (suppl. J. Phys. III (C3 5), 197-205.

Frost, H., Ashby, M., 1982. Deformation-mechanism maps. Pergamon Press, Oxford.

Gilormini, P., Nebozhyn, M.V., Castaeda, P.P., 2001. Accurate estimates for the creep behavior of hexagonal polycrystals. Acta Mater. 49, 329-337.

Goodman, D., Frost, H., Ashby, M., 1981. The plasticity of polycrystalline ice. Philos. Mag. 43, 665-695.

Greenland Ice core Project (GRIP) Members, 1993. Climate instability duringthe last interglacial period recorded in the grip ice core. Nature 364, 203-207.

Hamman, I., Weikusat, C., Azuma, N., Kipfstuhl, S., 2007. Evolution of crystal microstructure during creep experiments. J. Glaciol. 53, 479-489.

Humphreys, F.J., Hatherly, M., 1996. Recrystallization and Related Annealing Phenomena. Pergamon.

Jacka, T.H., Li,J., 1994. The steady-state crystal size of deforming ice. Ann. Glaciol. 20, 13-18.

Kipfstuhl, S., Hamann, I., Lambrecht, A., Freitag, J., Faria, S., Grigoriev, D., Azuma, N., 2006. Microstructure mapping: a new method for imaging deformation induced microstructural features of ice on the grain scale. J. Glaciol. 52, 398-406.

Kocks, U.F., Tom'e, C.N., Wenk, H., 1998. Texture and anisotropy. Preferred Orientations in Polycrystals and Their Effect on Materials Properties. Cambridge University Press0 521465168.

Lebensohn, R.A., 2001. N-site modeling of a 3d viscoplastic polycrystal using fast fourier transform. Acta Mater. 49, 2723-2737.

Lebensohn, R.A., Liu, Y., Ponte-Casta neda, P., 2004. On the accuracy of the selfconsistent approximation for polycrystals: comparison with full-field numerical simulations. Acta Mater. 52, 5347-5361.

Lebensohn, R.A., Montagnat, M., Mansuy, P., Duval, P., Meyssonnier, J., Philip, A., 2009. Modeling viscoplastic behavior and heterogeneous intracrystalline deformation of columnar ice polycrystals. Acta Mater. 57, 1405-1415.
Lebensohn, R.A., Tom'e, C.N., Ponte-Casta neda, P., 2007. Self-consistent modelling of the mechanical behaviour of viscoplastic polycrystals incorporating intragranular field fluctuations. Phil. Mag. 87, 4287-4322.

Lloyd, G., 1991. SEM electron channelling analysis of dynamic recrystallizationin a quartz grain. J. Struct. Geol. 13, 945-953.

Lloyd, G.E., Freeman, B., 1994. Dynamic recrystallization of quartz under greenschist conditions. J. Struct. Geol. 16, 867-881.

Mansuy, P., Meyssonnier, J., Philip, A., 2002. Localization of deformation in polycrystalline ice: experiments and numerical simulations with a simple grain model. Comput. Mater. Sci. 25, 142-150.

Mansuy, P., Philip, A., Meyssonnier, J., 2000. Identification of strain heterogeneities arising during deformation of ice. Ann. Glaciol. 30, 121-126.

Montagnat, M., Durand, G., Duval, P., 2009. Recrystallization processes in granular ice. Supp. Issue Low Temp. Sci. 68, 81-90.

Montagnat, M., Duval, P., 2000. Rate controlling processes in the creep of polar ice, influence of grain boundary migration associated with recrystallization. Earth Planet. Sci. 183, 179-186.

Montagnat, M., Duval, P., Bastie, P., Hamelin, B., Lipenkov, V.Y., 2003. Lattice distortion in ice crystals from the vostok core (antarctica) revealed by hard X-ray di_raction; implication in the deformation of ice at low stresses. Earth Planet. Sci. Lett. 214 369-378.

Montagnat, M., Weiss, J., Chevy, J., Duval, P., Brunjail, H., Bastie, P., Gil Sevillano, J., 2006. The heterogeneous nature of slip in ice single crystals deformed under torsion. Philos. Mag. 86, 4259-4270.

Moulinec, H., Suquet, P., 1998. A numerical method for computing the overall response of nonlinear composites with complex microstructure. Comput. Meth. Appl. Mech. Eng. 157, 69-94.

Nishikawa, O., Takeshita, T., 1999. Dynamic analysis and two types of kink bands in quartz veins deformed under subgreenschist conditions. Tectonophysics 301, 21-34.

Obbard, R., Baker, I., 2007. The microstructure of meteoric ice from Vostok, Antarctica. J. Glaciol. 53, 41-62.

Obbard, R., Baker, I., Sieg, K., 2006. Using electron backscatter di_raction patterns to examine recrystallization in polar ice sheets. J. Glaciol. 52, 546-557.

Orowan, E., 1942. A type of plastic deformation new in metals. Nature 149, 643-644.

Parrenin, F., Jouzel, J., Waelbroeck, C., Ritz, C., Barnola, J., 2001. Dating the Vostok ice core by an inverse method. J. Geophys. Res. 106, 31837-31851.

Piazolo, S., Borthwick, V., Griera, A., Montagnat, M., Jessell, M., Lebensohn, R., Evans, L., 2010. Substructure dynamics in minerals and metals: new insight from in-situ experiments, detailed EBSD analysis of experimental and natural samples and numerical modelling. Proceedings of ReX \& GG IV, pp. 34-40.

Piazolo, S., Montagnat, M., Blackford, J.R., 2008. Sub-structure characterization of experimentally and naturally deformed ice using cryo-ebsd. J. Microsc. 230, 509-519.

Ple, O., Meyssonnier, J., 1997. Preparation and preliminary study of structure controlled s2 columnar ice. J. Phys. Chem. B 101, 6118-6122.

Prior, D., 1999. Problems in determining the misorientation axes, for small angular misorientations, using electron backscatter di_raction in the sem. J. Microsc. 195, 217-225.

Prior, D.J., Wheeler, J., Peruzzo, L., Spiess, R., Storey, C., 2002. Some garnet microstructures: an illustration of the potential of orientation maps and misorientation analysis in microstructural studies. J. Struct. Geol. 24, 999-1011 Micro structural Processes: A Special Issue in Honor of the Career Contributions of R.H. Vernon. $\rightarrow$.

Reddy, S., Timms, N., Pantleon, W., Trimby, P.W., 2007. Quantitative characterization of plastic deformation of zircon and geological implications. Contrib. Mineralog. Petrol. 153, 625-645.

Russell-Head, D.S., Wilson, C., 2001. Automated fabric analyser system for quartz and ice. J. Glaciol. 24, 117-130.

Schulson, E.M., Duval, P., 2009. Creep and Fracture of Ice. Cambridge University Press.

Soula, A., Renollet, Y., Boivin, D., Pouchou, J.L., Locq, D., Caron, P., Y., Brchet, 2009. Analysis of high-temperature creep deformation in a polycrystalline nickel-base superalloy. Mater. Sci. Eng. A 510-511, 301-306.

Tommasi, A., Knoll, M., Vauchez, A., Signorelli, J., Thoraval, C., Log'e, R., 2009. Structural reactivation in plate tectonics controlled by olivine crystal anisotropy. Nat. Geosci. 2, 423-427.

Weikusat, I., Miyamoto, A., Faria, S.H., Kipfstuhl, S., Azuma, N., Hondoh, T., 2011 Subgrain boundaries in antarctic ice quantified by X-ray laue di_raction. J. Glaciol. 57, 111-120.

Wilson, C., Burg, J., Mitchell, J., 1986. The origin of kinks in polycrystalline ice Tectonophysics 127, 27-48.

Wilson, C., Zhang, Y., 1994. Comparison between experiment and computer modelling of plane strain simple shear ice deformation. J. Glaciol. 40, 46-55. 